\title{
The Control of Rate and Timing of Spikes in the Deep Cerebellar Nuclei by Inhibition
}

\author{
Volker Gauck and Dieter Jaeger \\ Department of Biology, Emory University, Atlanta, Georgia 30322
}

Cerebellar nucleus neurons were recorded in vitro, and dynamic clamping was used to simulate inhibitory synaptic input from Purkinje cells likely to occur in vivo. Inhibitory input patterns with varying synaptic amplitudes and synchronicity were applied to determine how spike rate and spike timing can be controlled by inhibition. The excitatory input conductance was held constant to isolate the effect of dynamic inhibitory inputs on spiking. We found that the timing of individual spikes was controlled precisely by short decreases in the inhibitory conductance that were the consequence of synchronization between many inputs. The spike rate of nucleus neurons was controlled in a linear way by the rate of inhibitory inputs. The spike rate, however, also depended strongly on the amount of synchronicity present in the inhibitory inputs. An irregular spike train similar to in vivo data resulted from applied synaptic conductances when the conductance was large enough to overcome intrinsic pacemaker currents. In this situation subthreshold fluctuations in membrane potential closely followed the time course of the combined reversal potential of excitation and inhibition. This indicates that the net synaptic driving force for realistic input levels in vivo may be small and that synaptic input may operate primarily by shunting. The accurate temporal control of output spiking by inhibitory input that can be achieved in this way in the deep cerebellar nuclei may be particularly important to allow fine temporal control of movement via inhibitory output from cerebellar cortex.

Key words: cerebellum; Purkinje cell; synaptic; coding; inhibition; dynamic clamp; in vitro; whole cell; synchronization
The final output of the cerebellum is generated by the neurons of the deep cerebellar nuclei (DCN). A major source of control over DCN neurons is derived from cerebellar cortex via GABA $_{A}$-type inhibitory Purkinje cell input (Billard et al., 1993). Purkinje cell synapses provide $73 \%$ of the total synapses to DCN neurons, and almost all somatic synapses of DCN neurons are inhibitory (Palkovits et al., 1977; De Zeeuw and Berrebi, 1995). This arrangement leads to the question of how inhibitory input can control spiking in postsynaptic neurons accurately. The required accuracy of this control appears to be quite high, because the function of the cerebellum has been related to precise temporal aspects of movement control (Braitenberg, 1967; Ivry and Keele, 1989; Diener et al., 1993; Braitenberg et al., 1997; Wang et al., 1998). For example, a recent study by Timmann et al. (1999) showed that throwing a ball accurately required a temporal precision of the coordination between arm movement and finger opening of $\sim 10 \mathrm{msec}$. Cerebellar patients were not able to throw accurately because the temporal precision was reduced. In the present study we investigated how the Purkinje cell projection onto the DCN might support such temporally precise control apparent from the behavioral level. Although the question of precise temporal coding of neural activity via excitatory input has been discussed extensively (Shadlen and Newsome, 1994, 1998; Softky, 1995; König et al., 1996), the possibility of such coding via inhibitory input has not been addressed to our knowledge.

To study how inhibitory Purkinje cell input might control DCN

Received Nov. 10, 1999; revised Feb. 2, 2000; accepted Feb. 4, 2000

This work was supported by the National Institute of Mental Health Grant MH-57256 and by Deutsche Forschungsgemeinschaft Research Fellowship GA$627-1$ to V.G.

Correspondence should be addressed to Dr. Dieter Jaeger, Department of Biology, 1510 Clifton Road, Emory University, Atlanta, GA 30322. E-mail: djaeger@emory.edu.

Copyright (C) 2000 Society for Neuroscience $\quad 0270-6474 / 00 / 203006-11 \$ 15.00 / 0$ spiking precisely, we used the technique of dynamic current clamping (Robinson and Kawai, 1993; Sharp et al., 1993). This method allowed us to record DCN neurons in vitro and to apply a conductance waveform that simulates the synaptic input of several hundred $\mathrm{GABA}_{\mathrm{A}}$-type input elements. This technique is distinctly different from directly injecting current waveforms because the current flowing through an applied synaptic conductance is dependent on the postsynaptic membrane potential. Furthermore, synaptic input conductances can change the temporal integration properties of neurons by reducing their input resistance and thereby their membrane time constant (Holmes and Woody, 1989; Rapp et al., 1992; Häusser and Clark, 1997). By applying simulated synaptic conductances to DCN neurons, we find that the rate and temporal precision of DCN spiking can be controlled by specific aspects of inhibitory input patterns.

\section{MATERIALS AND METHODS}

Tissue preparation and electrophysiology. All animal procedures fully complied with the National Institutes of Health guidelines on animal care and use. Whole-cell recordings were obtained at $32^{\circ} \mathrm{C}$ with an Axoclamp-2B amplifier from deep cerebellar nuclei neurons in $300 \mu \mathrm{m}$ sagittal cerebellar slices from 14- to 17-d-old male Sprague Dawley rats. The bridge was balanced and the voltage offset was zeroed before each recording. The slice medium contained (in $\mathrm{mm}$ ): $\mathrm{NaCl} 124, \mathrm{KCl} \mathrm{3}$, $\mathrm{KH}_{2} \mathrm{PO}_{4} 1.2, \mathrm{NaHCO}_{3} 26, \mathrm{CaCl}_{2} 2, \mathrm{MgSO}_{4} 1.9$, and glucose 20. Electrodes were filled with the following (in $\mathrm{mM}$ ): K-gluconate 140, HEPES $10, \mathrm{NaCl} 10$, EGTA 0.2, MgATP 4, NaGTP 0.4, spermine 0.05, glutathione 5, and $1 \%$ biocytin. Electrode resistance ranged between 6 and 12 $\mathrm{M} \Omega$. Excitatory and inhibitory synapses were blocked with $100 \mu \mathrm{M}$ AP-5, $10 \mu \mathrm{M} C N Q X$, and $40 \mu \mathrm{M}$ picrotoxin. Cells had to show a spike amplitude of at least $50 \mathrm{mV}$ during spontaneous activity and a drift of $<5 \mathrm{mV}$ in the mean membrane potential during the recording to be kept for data analysis. We did not subtract a junction potential from the recorded membrane potential. On theoretical grounds a junction potential of $\sim 10$ $\mathrm{mV}$ can be expected for recording solutions made with K-gluconate (Barry, 1994). This junction potential, however, can be primarily offset by a Donnan potential of opposite sign (Barry and Lynch, 1991). Neverthe- 
less, we cannot exclude some error in the absolute value of the recorded membrane potential. The results of the present study would not be affected by a small offset in the absolute membrane potential except for a matching shift in the described value of the combined synaptic reversal potential required to yield realistic spike rates.

Morphological characteristics of recorded cells. Cell bodies were visualized in the slice with a Zeiss $63 \times$ water immersion objective, and the image was captured on camera (Hamamatsu C2400) mounted on a $4 \times$ phototube and displayed on a monitor. The size of cell somata was clearly visible, and recordings were limited to large cells. Cells that appeared swollen, however, were also rejected. Recorded cells were labeled with biocytin, and the soma length and width of 15 out of 33 recorded neurons could be recovered after standard histological procedures. The length of the somata ranged from 20 to $34 \mu \mathrm{m}(26.1 \pm 5.1 \mu \mathrm{m}$, mean $\pm \mathrm{SD})$, and their width ranged from 12 to $23 \mu \mathrm{m}(16.3 \pm 3.1 \mu \mathrm{m}$, mean $\pm \mathrm{SD})$. GABAergic DCN neurons in rats have soma sizes between 5 and $22 \mu \mathrm{m}$ (mean, $10 \mu \mathrm{m}$ ), and glutamatergic DCN neurons have soma sizes between 10 and $35 \mu \mathrm{m}$ (mean, $20 \mu \mathrm{m}$ ). These distributions are almost identical for all three DCN (Batini et al., 1992). Because the DCN neurons in our experiments had soma sizes $>20 \mu \mathrm{m}$, it is very likely that we recorded mostly from glutamatergic DCN neurons that project to the red nucleus or thalamus. A small overlap with the largest of the inhibitory neurons projecting to the inferior olive, however, cannot be excluded. Dendritic arborizations were large and sparsely branched for all reconstructed cells without any indication of two distinct types. No physiological differences were observed between the smallest and the largest cells in our sample, and the data were pooled.

Simulated synaptic input. Dynamic clamping was used to inject a current $\left(I_{\mathrm{inj}}\right)$ at the cell soma that mimics the synaptic current of several hundred input neurons. The injected current was calculated during the recordings on the basis of the following equation: $I_{\mathrm{inj}}=\left(E_{\mathrm{ex}}-\mathrm{V}_{\mathrm{m}}\right) * G_{\mathrm{ex}}$ $+\left(E_{\mathrm{in}}-\mathrm{V}_{\mathrm{m}}\right) * G_{\mathrm{in}}$. The reversal potentials of the excitatory $\left(E_{\mathrm{ex}}\right)$ and the inhibitory $\left(E_{\mathrm{in}}\right)$ synaptic currents were set to 0 and $-70 \mathrm{mV}$, respectively. The recorded membrane potential $\left(\mathrm{V}_{\mathrm{m}}\right)$ and the injected current were updated with a frequency of $10 \mathrm{kHz}$. The excitatory and inhibitory conductances, denoted $G_{\text {ex }}$ and $G_{\text {in }}$, were calculated before the experiments. The excitatory conductance was always held at a constant value to isolate the effect of inhibitory input patterns on output spiking. A single inhibitory input element was simulated as a dual-exponential function with a rise time constant $\left(\tau_{1}\right)$ of $0.93 \mathrm{msec}$ and a decay time constant $\left(\tau_{2}\right)$ of 13.6 msec: $g_{\text {in }}=g_{\max } /\left(\tau_{2}-\tau_{1}\right) *\left(\mathrm{e}^{-(t / \tau 2)}-\mathrm{e}^{-(t / \tau 1)}\right)$. These time constants replicated experimental data of Anchisi et al. (1998). Our own measurements revealed almost identical values with a rise time constant of $1 \mathrm{msec}$ and a decay time constant of $13 \mathrm{msec}$ at a holding potential of $-40 \mathrm{mV}$ (data not shown). The parameter $g_{\max }$ has not been determined experimentally yet, and we used different gain factors to explore a realistic range of input conductance amplitudes. A $g_{\max }$ of $4.3 \mathrm{pS}$ corresponds to a gain factor of 1 in our experiments. The applied inhibitory conductance trace $\left(G_{\text {in }}\right)$ was the sum of all inhibitory input conductances over time. It has been estimated that on average 860 Purkinje cells converge onto each nucleus neuron (Palkovits et al., 1977). Purkinje cell synapses are located to $12 \%$ (103) on the soma and to $37 \%$ (318) on the proximal dendrites of nucleus neurons (De Zeeuw and Berrebi, 1995). For our dynamic-clamp stimuli, we chose 400 inhibitory input elements because this number approximates the sum of the Purkinje cells that contact each nucleus neuron electrotonically close to its spike initiation zone. Purkinje cells in vivo show an ongoing activity with mean frequencies of $35 \mathrm{~Hz}$ (Savio and Tempia, 1985; Stratton et al., 1988), which we used as the mean frequency of our input elements. The interspike intervals of the input elements were generated randomly (exponential distribution). The resulting amplitude of $G_{\text {in }}$ had a mean of $1 \mathrm{nS}$ at an input gain of 1. The amplitude of $G_{\text {ex }}$ was set so that the mean value of the combined excitatory and inhibitory synaptic reversal potential $\left(\mathrm{V}_{\text {syn }}\right)$ was close to $-40 \mathrm{mV}: \mathrm{V}_{\mathrm{syn}}=\left(E_{\mathrm{ex}} * G_{\mathrm{ex}}+E_{\mathrm{in}} * G_{\mathrm{in}}\right) /\left(G_{\mathrm{ex}}+G_{\mathrm{in}}\right)$. This value was required to result in realistic output spike frequencies. Using the equation describing $\mathrm{V}_{\text {syn }}$, we can express the injected current as: $I_{\text {inj }}$ $=\left(G_{\mathrm{ex}}+G_{\mathrm{in}}\right) *\left(\mathrm{~V}_{\mathrm{syn}}-\mathrm{V}_{\mathrm{m}}\right)$, showing that $I_{\mathrm{inj}}$ is proportional to the total input conductance and the deviation of the synaptic reversal potential from the membrane potential. For technical details of the dynamic current-clamp experiments, see Jaeger and Bower (1999).

Analysis of the spike-time precision. Each simulated synaptic input pattern lasted $5 \mathrm{sec}$ and was applied repeatedly (two to eight times) with pauses of $5 \mathrm{sec}$ in between. The cross-correlogram was calculated from the spike times of successive spike trains with a bin width of 1 msec. The spike-timing precision was defined as the percentage of the spikes that did fall into time windows of \pm 1 and $\pm 5 \mathrm{msec}$ in the cross-correlogram. The chance level was subtracted from these values. To calculate the chance level, we randomly redistributed (shuffled) the interspike intervals and calculated the cross-correlogram from the shuffled spike trains. The subtracted chance level depended on the spike frequency. For example, at a mean spike rate of $20 \mathrm{~Hz}$ and a precision criterion of \pm 5 msec, the subtracted chance level was $20 \%$, and therefore the maximal proportion of precisely timed spikes was limited to $80 \%$.

Spike-triggered averaging and reconstruction of input conductance. Spike-triggered averages (STAs) of normalized input frequency and input conductance were computed for a duration of $\pm 100 \mathrm{msec}$ around the peak of action potentials. To obtain STAs of the input frequency, we convolved each input spike with a $1 \mathrm{msec}$ Gaussian of a peak amplitude of 1.0. This operation leads to an analog trace of the instantaneous spike rate (Paulin, 1996). The STA was computed after normalizing the traces to a mean of 1.0. To reconstruct the estimate of the input conductance on the basis of its STA, we replaced each output spike from all presentations of the same stimulus with its STA and added up the STAs. The resulting trace was divided by the number of stimulus repetitions.

\section{RESULTS}

\section{Spontaneous activity in current clamp}

All recorded DCN neurons were spontaneously active when synaptic input was blocked, and no bias current was injected. Their mean spontaneous spike frequency was $7.7 \pm 4.2 \mathrm{~Hz}(n=$ 33). The corresponding mean value of the subthreshold membrane potential was $-49.4 \pm 3.0 \mathrm{mV}$. The voltage from $3 \mathrm{msec}$ before to $3 \mathrm{msec}$ after the peak of each action potential was removed to obtain this value. Most of the recorded neurons were regular spiking $(82 \%)$, whereas the remaining neurons showed some tendency of spontaneous bursting. These characteristics were similar to those seen previously (Jahnsen, 1986a; Aizenman et al., 1998). No differences between regular spiking and bursting neurons were observed in the dynamic-clamp experiments, and data from both groups were pooled.

\section{Inhibitory synaptic input can control the spike timing of DCN neurons precisely}

We used the activity of 400 simulated presynaptic Purkinje cell elements to construct an inhibitory conductance trace (Fig. $1 \mathrm{~A}$, $\left.G_{\text {in }}\right)$. This conductance trace presents the sum of all unitary postsynaptic conductance changes caused by individual presynaptic elements. The excitatory input conductance was held constant throughout this study to examine the effects of inhibitory input patterns in isolation (Fig. $1 A, G_{\mathrm{ex}}$ ). The amplitude of the constant excitatory conductance was chosen so that the combined synaptic reversal potential of inhibition and excitation $\left(\mathrm{V}_{\mathrm{syn}}\right)$ had a mean value of $-40 \mathrm{mV}$ (Fig. $1 B$, gray trace). Because the reversal potential of the inhibitory conductance $\left(E_{\text {in }}\right)$ was -70 $\mathrm{mV}$ and that of the excitatory conductance $\left(E_{\text {ex }}\right)$ was $0 \mathrm{mV}$, the amplitude of the mean excitatory conductance needed to be $75 \%$ that of the mean inhibitory conductance. $\mathrm{V}_{\mathrm{syn}}$ can be computed from the inhibitory and excitatory conductances as: $\mathrm{V}_{\mathrm{syn}}=\left(E_{\mathrm{ex}} *\right.$ $\left.G_{\mathrm{ex}}+E_{\mathrm{in}} * G_{\mathrm{in}}\right) /\left(G_{\mathrm{ex}}+G_{\mathrm{in}}\right)$. The membrane potential trajectory (Fig. $1 B$, black trace) followed fluctuations in $\mathrm{V}_{\mathrm{syn}}$ quite well. These fluctuations reflect the changing amplitude of the inhibitory conductance. During depolarizing membrane potential fluctuations, action potentials could be generated. There was not a fixed voltage threshold, however. In particular, spikes that followed a preceding spike at a short interval showed a higher threshold (Fig. 1B, asterisk). This effect is likely caused by a remaining sodium channel inactivation after the preceding spike.

Changes in membrane potential attributable to synaptic input are the consequence of the current injected across the synaptic conductance. The general equation for injected current $\left(I_{\text {inj }}\right)$ via 
A: conductances

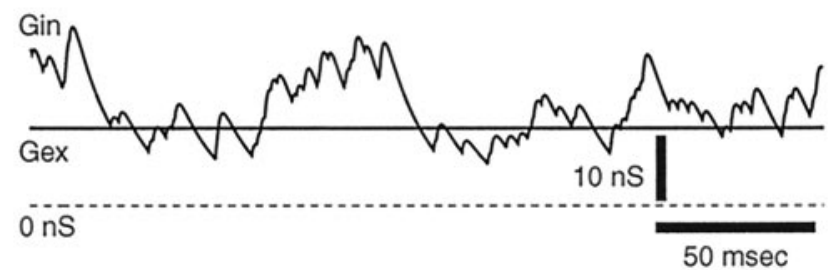

B: Vm (black) and Vsyn (gray)

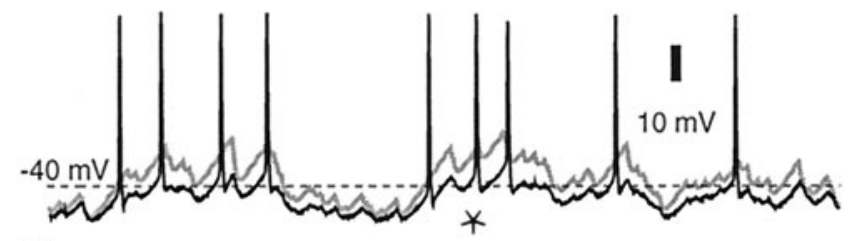

C: injected current

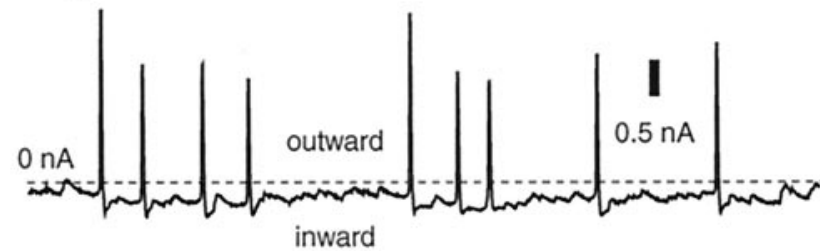

D: spike raster

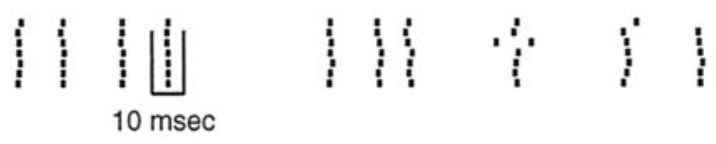

Figure 1. Spike timing of DCN neurons can be controlled precisely by inhibitory input. $A$, The temporal modulation of the inhibitory conductance $\left(G_{\text {in }}\right)$ was determined by the pattern of inhibitory inputs. The excitatory conductance $\left(G_{\mathrm{ex}}\right)$ was constant. The mean values of $G_{\mathrm{ex}}$ and $G_{\text {in }}$ were 12 and $16 \mathrm{nS}$, respectively. The stimulus conditions corresponded to a gain factor of 16 and a high input synchronization (see Fig. 2). $B$, The subthreshold membrane potential $\left(\mathrm{V}_{\mathrm{m}}\right.$, black trace $)$ followed the combined synaptic reversal potential $\left(\mathrm{V}_{\mathrm{syn}}\right.$, gray trace $)$. The mean value of $\mathrm{V}_{\text {syn }}$ was $-40 \mathrm{mV}$. C, The injected current $\left(I_{\text {inj }}\right)$ was determined by the difference between $\mathrm{V}_{\mathrm{m}}$ and $\mathrm{V}_{\mathrm{syn}}$ and by the total synaptic strength $\left(G_{\mathrm{ex}}+\right.$ $\left.G_{\text {in }}\right)$ (see Materials and Methods). $D$, The stimulus-aligned spike raster recorded from one DCN neuron to six stimulus repetitions illustrates the millisecond precision in the alignment of spikes to the synaptic input.

a mix of excitatory and inhibitory synaptic conductances is: $I_{\mathrm{inj}}=$ $\left(G_{\mathrm{ex}}+G_{\mathrm{in}}\right) *\left(\mathrm{~V}_{\mathrm{syn}}-\mathrm{V}_{\mathrm{m}}\right)$. It is very important to notice that $I_{\mathrm{inj}}$ is exactly zero when the membrane potential equals $\mathrm{V}_{\text {syn }}$. For any deviation of $\mathrm{V}_{\mathrm{m}}$ from $\mathrm{V}_{\text {syn }}$ a synaptic current is injected that drives $\mathrm{V}_{\mathrm{m}}$ back toward $\mathrm{V}_{\text {syn }}$. The larger the total synaptic conductance $\left(G_{\mathrm{ex}}+G_{\mathrm{in}}\right)$, the larger is the synaptic current that stabilizes $\mathrm{V}_{\mathrm{m}}$ at $\mathrm{V}_{\text {syn }}$. Thus $\mathrm{V}_{\text {syn }}$ can be seen as the command voltage of a partial voltage clamp operating at a gain proportional to $G_{\mathrm{ex}}+G_{\mathrm{in}}$. The action of mixed excitatory and inhibitory conductances as a partial voltage clamp is in principle a generalization of the classical concept of shunting. The term shunting is used to describe the case in which an open inhibitory conductance stabilizes $\mathrm{V}_{\mathrm{m}}$ near the inhibitory reversal potential. We ascribe the term partial voltage clamp to the situation in which a mix of open excitatory and inhibitory conductances stabilizes $\mathrm{V}_{\mathrm{m}}$ near the combined reversal potential. Figure $1, B$ and $C$, shows that $\mathrm{V}_{\mathrm{m}}$ stayed very close to $\mathrm{V}_{\text {syn }}$ except during an action potential. Therefore the synaptic input operated quite effectively as a voltage clamp. The injected synaptic current was on average a small depolarizing current, indicating that the intrinsic currents of the neuron had a small outward bias at $-40 \mathrm{mV}$, which the synaptic clamp current counteracted. During an action potential the neuron depolarized rapidly, and the clamp current showed a hyperpolarizing current peak that counteracted the intrinsic inward current.

All recorded DCN neurons $(n=33)$ showed a high reliability in the response to repetitions of the same stimulus (Fig. 1D). This finding indicates that inhibitory input patterns can control output spiking precisely. The specific stimulus conditions that control spike timing and spike frequency are examined in the following sections.

\section{Spike-timing precision increased with input gain and input correlation}

An important mechanism by which cerebellar cortex may convey information to the DCN lies in the degree of synchronicity in the activity of many Purkinje cells converging onto DCN neurons. Climbing-fiber inputs for example may lead to highly synchronous activation of a population of Purkinje cells (Welsh et al., 1995; Lang et al., 1999). We tested the effects of input synchronicity on DCN spiking by using three different synchronization levels. At the highest level of input synchronization, our 400 presynaptic elements were divided into 10 groups of 40 synchronously activated elements. The activity between groups was uncorrelated. An intermediate level of input synchronization consisted of 100 groups of 4 synchronized elements. In the condition without input synchronization, all 400 input elements were activated independently.

A second parameter that we manipulated was the total amplitude of $G_{\text {in }}$ plus $G_{\text {ex }}$ by multiplying both $G_{\text {in }}$ and $G_{\text {ex }}$ with the same gain factor. This manipulation does not change the time course of $\mathrm{V}_{\text {syn }}$, but the amount of synaptic current for a given offset between $\mathrm{V}_{\text {syn }}$ and $\mathrm{V}_{\mathrm{m}}$ is proportional to this gain factor. The use of a range of different synaptic strengths was important because the precise value of this parameter in vivo has not yet been determined.

We found that both the level of input synchronicity and the level of synaptic strength (input gain) had important consequences for the control of DCN spiking by inhibitory input (Fig. 2). The accuracy of the temporal alignment of spikes with the stimulus was increased by a rise of the input gain as well as by a rise of the input synchronization (Fig. $2 A, B$ ). The lowest input gain used was ineffective in controlling spike timing as demonstrated by the absence of spike alignment in the spike rasters and the absence of a central peak in the cross-correlation of spike trains between subsequent stimulus presentations (Fig. 2A,B). At the highest input gain and high input synchronicity, $62 \%$ of spikes were aligned to the stimulus with a $\pm 5 \mathrm{msec}$ precision, and $48 \%$ of spikes were aligned even with a $\pm 1 \mathrm{msec}$ precision (Fig. $2 C, D$; mean values of 21 cells). Lowering the synchronization to the intermediate level but remaining at a high gain resulted in a partial loss of spike-timing precision. The decrease of spike precision with a decrease in input gain was gradual, and the presence of $\pm 1 \mathrm{msec}$ precision was lost before the $\pm 5 \mathrm{msec}$ criterion, was also not reached at very low gains (Fig. 2C,D).

The input synchronization of presynaptic elements in several independent groups may misrepresent the possible case of just one synchronous group in the input to a given DCN neuron. To verify this case we used an input condition in which only one group of inhibitory input elements was synchronized while all remaining input elements were independently active. The num- 
A: high input synchronization

gain factor: 0.5

$\because \because \because \because \because \because \because \because$ :

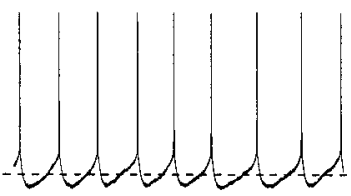

$-50 \mathrm{mv}$

cross-correlation

0.2 events/s

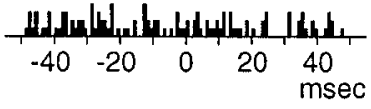

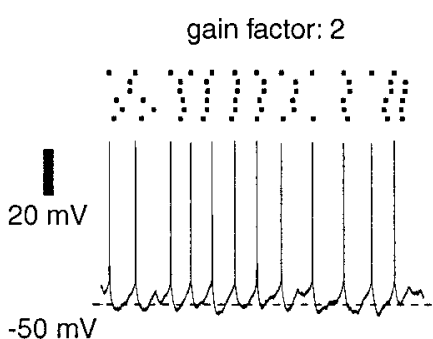

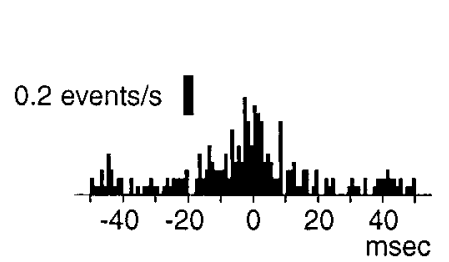

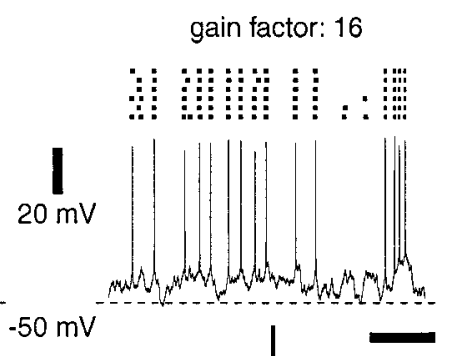

$200 \mathrm{msec}$

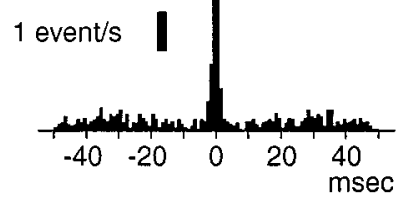

B: intermediate input synchronization gain factor: 0.5

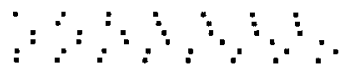

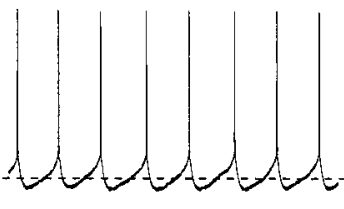
cross-correlation 0.2 events/s

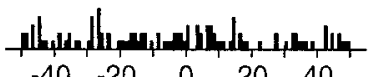
$\begin{array}{lllll}-40 & -20 & 0 & 20 & 40 \\ \mathrm{msec}\end{array}$ gain factor: 2
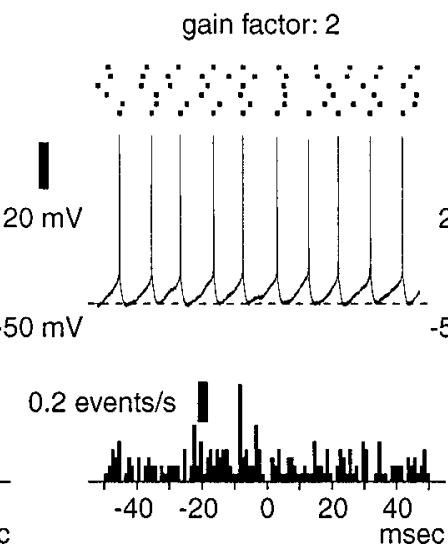
gain factor: 16
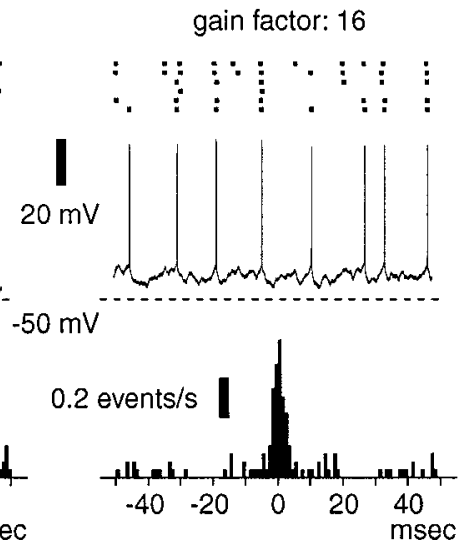

C: precision criterion: $\pm 5 \mathrm{msec}$

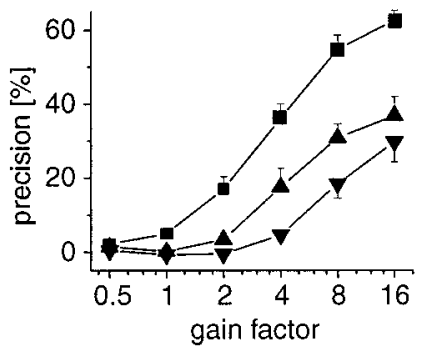

D: precision criterion: $\pm 1 \mathrm{msec}$

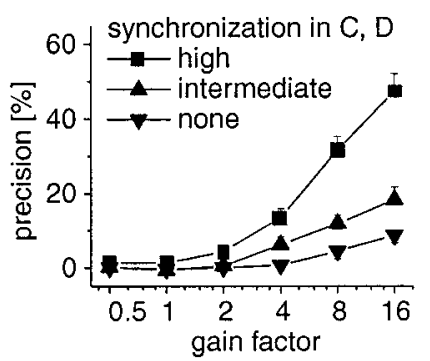

$\mathrm{E}$ : precision criterion: $\pm 5 \mathrm{msec}$

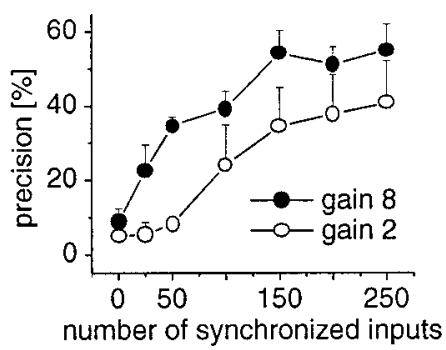

Figure 2. Spike-timing precision increased with input gain and input synchronization. $A$, Stimulus-aligned spike rasters, voltage traces, and crosscorrelograms of one DCN neuron at a high level of input synchronization. At the input gain of 16, the percentage of all spikes that were precisely aligned within a window of $\pm 5 \mathrm{msec}$ in the cross-correlogram of this neuron was $67 \%$ (shuffle corrected). $B$, Spike rasters, voltage traces, and cross-correlograms of the same neuron when an intermediate input synchronization was used. The proportion of precisely timed spikes ( $\pm 5 \mathrm{msec})$ was reduced to $35 \%$ at a gain of 16. $C$, Average spike-timing precision of recorded DCN neurons (mean \pm SE) as a function of the input gain and synchronization. Numbers of cells averaged were 19 for high, 12 for intermediate, and 12 without input synchronization. The precision criterion is the shuffle-corrected percentage of spikes that fell into a time window of $\pm 5 \mathrm{msec}$ in the cross-correlogram (see Materials and Methods). $D$, Same data as in $C$ for a different precision criterion $( \pm 1 \mathrm{msec})$. $E$, Spike-timing precision (criterion, $\pm 5 \mathrm{msec}$ ) as a function of the number of synchronized input elements at an input gain of 2 and $8(n=6)$. Note that in $E$ input elements were synchronized only within one group in contrast to the synchronization in $A-D$.

ber of synchronized elements was systematically increased $(0,25$, $50,100,150,200$, and 250) for two different input gain factors (2 and 8 ). The findings generally mirrored the case of multiple synchronized input groups. The precision increased with the input synchronization and with the input gain (Fig. 2E). At an input gain of $8, \sim 150$ of 400 input elements needed to be synchronized to reach a proportion of $55 \%$ of spikes timed within \pm 5 msec in the cross-correlation (Fig. $2 E$ ), a precision corresponding to that for the high input synchronization at a gain of 8 in Figure $2 C$. At an input gain of 2, 100 of 400 input elements had to be synchronized to reach a spike timing precision of $20 \%( \pm 5 \mathrm{msec})$ that corresponds to the precision for the high input synchronization at a gain of 2 in Figure $2 C$.

\section{Increasing input gain transformed a regular into an irregular spike pattern}

DCN neurons in vivo show a highly irregular spiking pattern (Thach, 1968; LeDoux et al., 1998). In contrast, the spontaneous spiking of most DCN neurons in vitro is very regular (Jahnsen, 1986a; Aizenman and Linden, 1999). A likely cause of this difference is the presence of network activity and patterned synaptic input in vivo. To determine the relationship between the presence of synaptic input and the spiking pattern, we examined how interspike interval (ISI) distributions and autocorrelograms (ACs) varied as a function of an increasing level of synaptic input. At the lowest input gain, DCN neurons showed a regular firing pattern (Fig. $2 A, B$ ) very similar to their spontaneous spiking in the absence of any input. Regular spiking was reflected in a narrow symmetrical ISI distribution and a periodic AC (Fig. $3 A, B)$. In contrast, at a high input gain and a high input synchronization, the firing pattern was highly irregular (Fig. $2 A$ ). Therefore, the ISI distribution was broad and asymmetric, and the AC showed no periodicity (Fig. $3 A$ ). At a high input gain but an intermediate input synchronization, the neurons also showed an irregular firing pattern (Figs. $2 B, 3 B$ ) and a nonperiodic AC (Fig. $3 B)$. The ISI distribution at the intermediate input synchronization, however, lacked the distinct peak of short ISIs present with 


\section{A: high input synchronization}

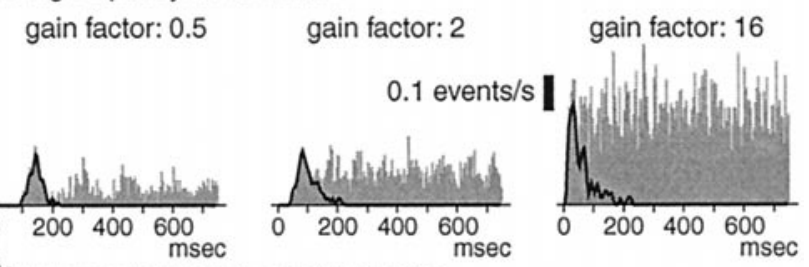

B: intermediate input synchronization

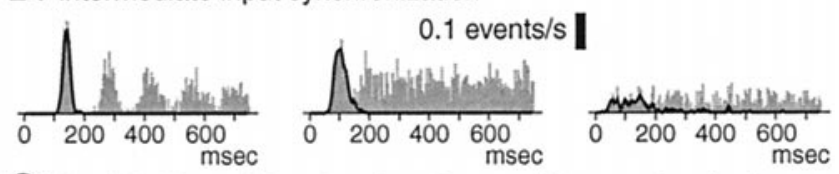

$\mathrm{C}$ : Vm (black) and Vsyn (gray) at intermediate synchronization

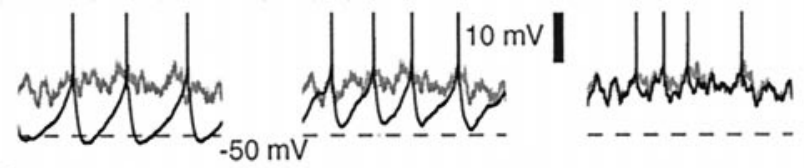

D: injected current at intermediate synchronization

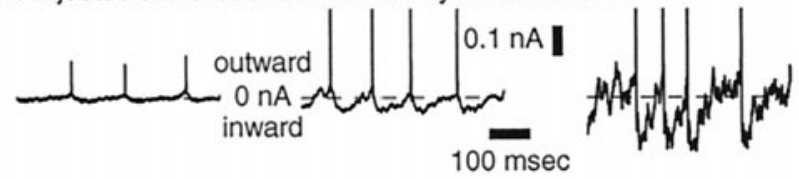

Figure 3. The irregularity of the spike pattern increased with the partial voltage-clamp effect of the synaptic input. $A$, Autocorrelograms (gray vertical bars) and interspike interval distributions (black lines) at high input synchronization are shown. $B$, Autocorrelograms ( gray vertical bars) and interspike interval distributions (black lines) are shown. The plots are the same as in $A$ but for intermediate input synchronization. $C, \mathrm{~V}_{\mathrm{m}}$ (black trace) followed $\mathrm{V}_{\text {syn }}$ (gray trace) more closely the larger the input gain was. The neuron and the input gains and synchronization are the same as in $B$. Action potentials are cut off at $-25 \mathrm{mV}$ to expand the subthreshold potential range. $D$, The traces of $I_{\text {inj }}$ corresponding to the voltage traces in $C$ are shown. An increasing input gain caused a larger synaptic current that clamped $\mathrm{V}_{\mathrm{m}}$ more effectively to $\mathrm{V}_{\mathrm{syn}}$. The applied outward current was cut off at $0.3 \mathrm{nA}$.

high input synchronization (Fig. $3 A, B$ ). This difference is similar to the different ISI distributions of DCN neurons reported in awake monkeys in which the ISI distribution during arm movement showed a large peak that was absent without movement (Thach, 1968). Our results suggest that these differences may be caused by a higher amount of synchronicity in the Purkinje cell input during arm movement. None of the ISI distributions observed in vivo corresponded to our case of low input gain.

An increase in synaptic gain leads to an increase in spiking irregularity as well as an increase in spike-time precision. The mechanism underlying these two effects is most easily understood by the voltage-clamping effect of synaptic conductances. When no input or input at a very low gain was given, cells showed stimulusindependent pacemaker activity (Fig. 3C, black trace). The synaptic conductance was not large enough to result in sufficient current (Fig. 3D) to pull the membrane potential close to $\mathrm{V}_{\text {syn }}$ (Fig. 3C, gray trace). When the input gain was increased, cells were forced to follow $\mathrm{V}_{\text {syn }}$ more closely (Fig. 3C,D). In particular, the simulated synaptic current was now of sufficient amplitude to counteract the intrinsic hyperpolarizing current after each spike. This can be seen as a depolarizing current after each spike (Fig. $3 D$ ). Approximately $50 \mathrm{msec}$ after each spike, $\mathrm{V}_{\mathrm{m}}$ started to track $\mathrm{V}_{\text {syn }}$ quite closely, and the simulated synaptic current was near zero. Spike timing in this condition was precisely aligned to the stimulus, because threshold crossings were determined by the trajectory of $\mathrm{V}_{\mathrm{syn}}$. The spike pattern was irregular because fluctuations in $\mathrm{V}_{\text {syn }}$ of sufficient size to trigger a spike occurred at irregular intervals.

The number and location of Purkinje cell synapses onto DCN neurons (Palkovits et al., 1977; De Zeeuw and Berrebi, 1995) and the mean rate of Purkinje cell activity (Savio and Tempia, 1985; Stratton et al., 1988) are fairly well established and were approximated by our dynamic-clamp stimuli (see Materials and Methods). The amplitude of unitary postsynaptic conductances of Purkinje cell inputs has not been determined yet. Our data result in an approximate estimate of a possible range of unitary synaptic conductances of Purkinje cell inputs. We found that a total mean inhibitory conductance of at least $8 \mathrm{nS}$ was required so that inhibition could control DCN spiking. This corresponds to a unitary conductance change with a peak value of $34 \mathrm{pS}$ resulting in a postsynaptic peak current of $1 \mathrm{pA}$ at a driving force of $30 \mathrm{mV}$. This is still a very small value for a synaptic conductance, indicating that the real value might be larger. Preliminary data with minimal electrical stimulation of Purkinje cell inputs in our lab suggest unitary synaptic currents of $10 \mathrm{pA}$ or below. These considerations indicate that individual Purkinje cells have only a small influence on single DCN neurons but that the voltageclamp gain of the total synaptic input in vivo is likely to be significant.

\section{Spikes were triggered by transient decreases in inhibition}

To understand how inhibitory input is coded in output spiking, it is important to determine which particular input patterns trigger spikes and how a precise timing relation to the input is achieved. To analyze what input events led to spike generation, we calculated STAs of the inhibitory input frequency and of the inhibitory input conductance (Fig. 4A,B). STAs correspond to the first Wiener kernel of a stimulus reconstruction and frequently capture most of the information about the input present in the output spike times (Bialek and Rieke, 1992). We found that spikes were preceded by a transient decrease in input frequency that was most pronounced at a high input gain and high input synchronization (Fig. 4A). This decrease in frequency started $\sim 18 \mathrm{msec}$ before the output spike and abruptly ended right at the time of the output spike. As expected, there was no consistent stimulus waveform surrounding a spike at low input gains, because spikes were not aligned to the stimulus under these conditions (Fig. $2 A, B)$. A reduction in input synchronization resulted in a reduced amplitude of the spike-triggered average, whereas the onset time of the reduction was not changed (Fig. $4 A$ ).

The STA of the input conductance (Fig. $4 B$ ) is closely related to the STA of the input frequency in that each input spike contributes a unitary postsynaptic conductance change. Because the conductance change follows an input spike and has a decay time constant of $13 \mathrm{msec}$, the spike-triggered conductance change has a slightly delayed onset and a longer duration compared with the change in the input frequency. We reconstructed the expected inhibitory input conductance using our spike trains by convolving each output spike with the STA of the conductance (Fig. 4C,D). The reconstruction showed that the output spikes contain reliable information about the occurrence of transient decreases in the inhibitory input. In contrast, increases in inhibition from the mean value were not at all coded in the output spikes. In addition, cells clearly did not preferentially respond to events consisting of releases in inhibition from increased levels to the mean level (Fig. 


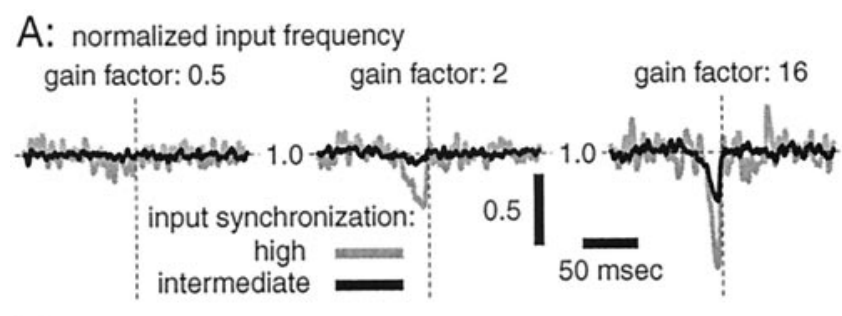

B: normalized inhibitory conductance

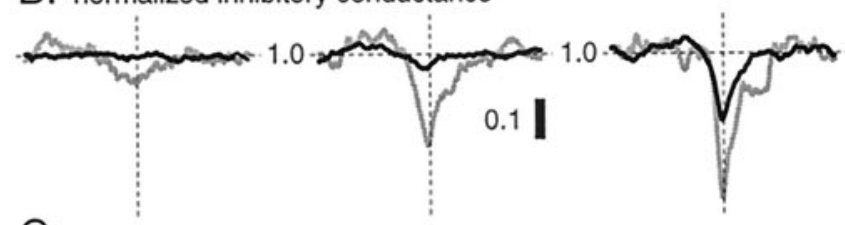

C: spike raster high input synchronization

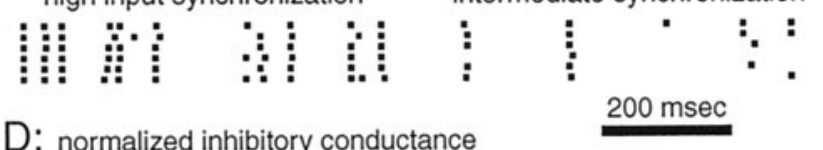

D: normalized inhibitory conductance

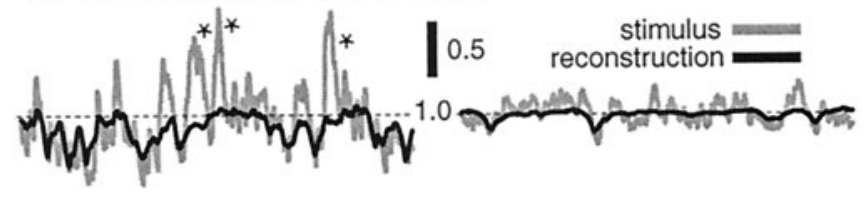

Figure 4. Spikes were triggered by short decreases in inhibition. $A$, STAs of the normalized inhibitory input frequency. The value of 1.0 corresponds to the mean input frequency of $35 \mathrm{~Hz}$. The instantaneous input frequencies were computed by convolving each input spike with a $1 \mathrm{msec}$ Gaussian. The input synchronization was high (gray trace) or intermediate (black trace). $B$, STAs of $G_{\text {in }}$ normalized to the mean level of conductance at each input gain. The value of 1.0 corresponds to $0.5 \mathrm{nS}$ (left), $2 \mathrm{nS}$ (middle), and $16 \mathrm{nS}$ (right). The neuron, input synchronizations, and input gains are the same as in $A$. $C$, Stimulus-aligned spike rasters for two input synchronizations for the input gain factor of 16 . The neuron is the same as in $A$ and $B . D, G_{\text {in }}$ (gray trace) and the reconstruction of $G_{\text {in }}$ (black trace) calculated by convolving the spike rasters in $C$ with the corresponding STAs in $B$.

$4 C, D$, asterisks). Such a response would indicate the presence of postinhibitory rebound spiking. Although such rebound spiking was elicited in our recordings after negative current injection pulses in vitro as observed previously (Jahnsen, 1986a; Aizenman et al., 1998), the stimulus condition of ongoing excitatory and inhibitory input conductances did not trigger this response. This can be explained by the fact that the combined reversal potential of inhibition and excitation $\left(\mathrm{V}_{\mathrm{syn}}\right)$ and thus $\mathrm{V}_{\mathrm{m}}$ did not reach values negative to $-60 \mathrm{mV}$ to deinactivate the channels required for the rebound response.

The input sequences we selected were aimed to simulate possible patterns of Purkinje cell input to DCN neurons. Because only white-noise stimuli yield STAs that are unbiased by the stimulus (Rieke et al., 1996), our STAs may reflect stimulus properties such as the time course of unitary IPSCs. Stimuli with different properties such as gain and synchronicity, however, showed STAs with a nearly identical time course but a different amplitude. We also constructed a stimulus, in which we synchronized pauses $(10$ or $50 \mathrm{msec}$ ) of spiking between groups of input elements but not the occurrence of spikes. The STA of the conductance for this stimulus was also very similar (data not shown). The stimulus condition of just one single group of synchronized input elements briefly described above was a special case because in this situation each decrease in inhibition was preceded by an increase relative to the mean. Thus the STA of this stimulus also showed a peak preceding the decrease in $G_{\text {in }}$ that triggered a spike (data not shown). Overall these data show that for a wide range of presynaptic input patterns the spiking of DCN neurons was reliably triggered by decreases in the inhibitory input conductance that showed a minimum duration of $\sim 15 \mathrm{msec}$.

\section{The precision of spike timing stayed high when synchronized inputs were jittered}

Perfect input synchronization as used above is probably not usually achieved in vivo. To examine the effects of decreasing precision in the presynaptic synchronization, we constructed a set of stimuli with jitter. Using a stimulus with high gain (factor 8) and high synchronization, we systematically decreased the input synchronization by jittering the input spikes. The jitter value used for each spike was random within a range of $5 \mathrm{msec}( \pm 2.5 \mathrm{msec})$, $10 \mathrm{msec}$ ( $\pm 5 \mathrm{msec}), 20 \mathrm{msec}( \pm 10 \mathrm{msec})$, or $50 \mathrm{msec}( \pm 25 \mathrm{msec})$. The resulting alignment of DCN output spikes with the stimulus stayed high for most jitter conditions (Fig. $5 A$ ). For the precision criterion of $\pm 5 \mathrm{msec}$, the proportion of stimulus-aligned spikes stayed nearly constant up to a jitter of $20 \mathrm{msec}$ (Fig. 5C). For the more stringent precision criterion of $\pm 1 \mathrm{msec}$, the number of well aligned spikes started to fall off markedly at a jitter of $5 \mathrm{msec}$ (Fig. $5 C$ ). Even at a jitter of $50 \mathrm{msec}$ the stimulus alignment of spikes was better compared with the reference case in which all input elements were independently active (Fig. 2C,D). The maintained output precision despite input jitter can be explained by the averaging effect resulting from jittering many spikes independently. In essence this manipulation corresponds to a low-pass filtering of the synaptic conductance trace $G_{\text {in }}$ (Fig. $5 B$ ). Physiologically this finding signifies that the convergence of many presynaptic inhibitory elements allows for the precise control of output spiking even when the input events are not aligned as precisely. A similar function of sharpening the timing of output spikes by large sets of less well aligned input spikes has been put forth for excitatory inputs in the so-called synfire chain hypothesis (Abeles, 1991).

\section{The spike rate was controlled by the rate of inhibitory input, whereas the spike precision remained unaffected}

Spike-rate coding is often considered to be the most important mechanism of information transfer between neurons in the brain. In a spike-rate code the frequency of the synaptic inputs over some time window controls the frequency of output spiking. There is ample evidence in the literature that Purkinje cells show changes in spike rate related to behavior for time periods of 10-500 msec (Mano et al., 1991; Fortier et al., 1993; Krauzlis and Lisberger, 1996). The baseline spiking frequency in awake rats is $\sim 35 \mathrm{~Hz}$ (Savio and Tempia, 1985; Stratton et al., 1988), which we used as the mean frequency of our inhibitory input elements for the stimuli described above. To determine how frequency changes in the inhibitory input to DCN neurons affects output spiking, we tested one set of cells $(n=5)$ for five different inhibitory input frequencies. The effect of input frequency was tested at two input gains (factor 2 and 8 ) with high or without input synchronization. We found that under all stimulus conditions the output frequency increased with decreases in the inhibitory input frequency (Fig. $6 A, D)$. The average membrane potential after removal of spikes was more depolarized at high output frequencies (Fig. $6 E$ ), reflecting the depolarization in $\mathrm{V}_{\text {syn }}$ with decreases in inhibitory input. In general, a linear relationship was found between the 


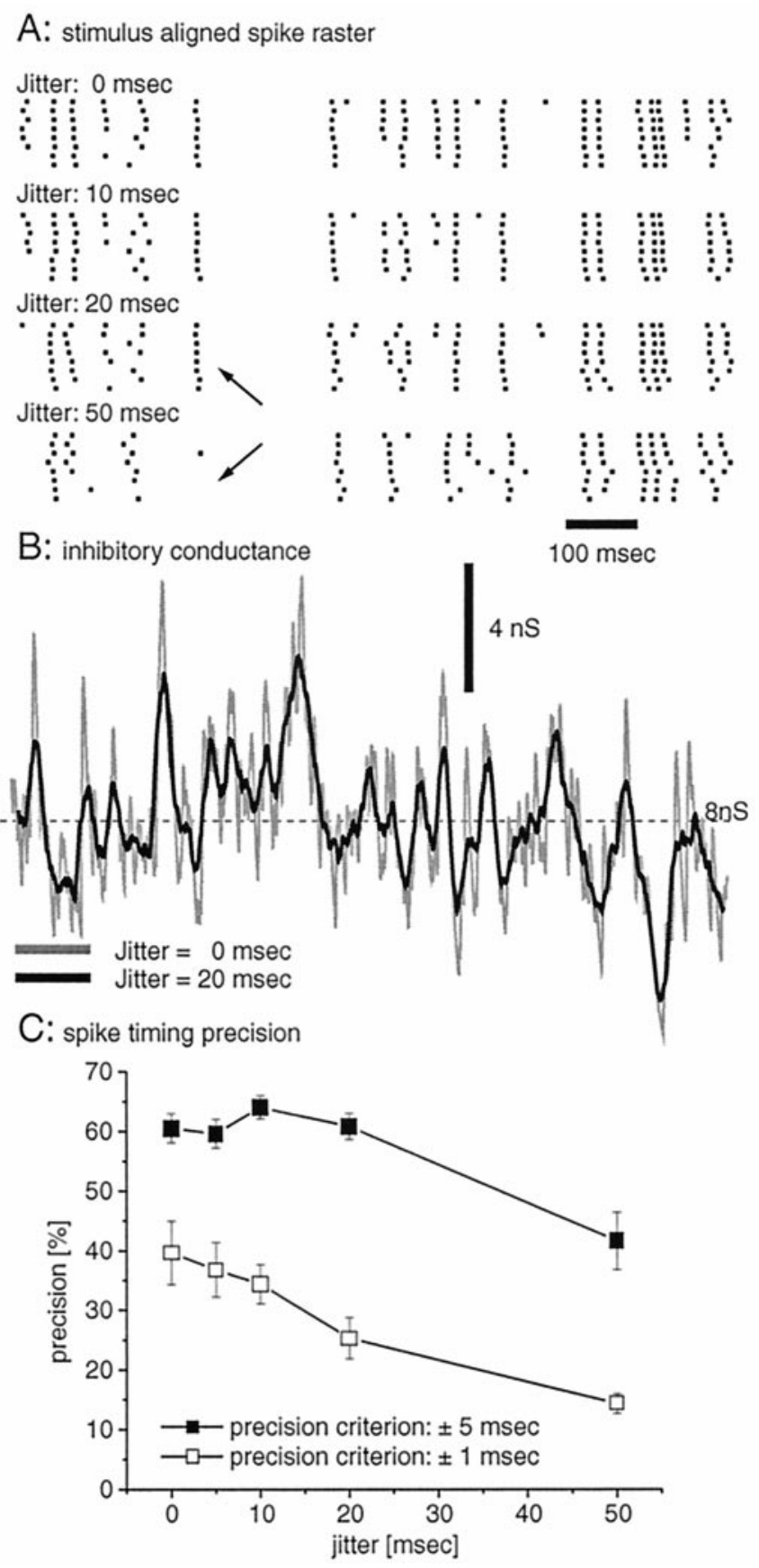

Figure 5. The spike timing was robust against temporal jitter in the input. $A$, Stimulus-aligned spike rasters of one DCN neuron for four values of temporal jitter at an input gain of 8 . The input synchronization was high. Some spikes disappeared at the largest jitter (arrows). B, Inhibitory conductance traces without jitter (gray) and with $20 \mathrm{msec}$ of jitter (black). C, Spike-timing precision of five DCN neurons (mean \pm $\mathrm{SE}$ ) as a function of jitter for the same input condition shown in $A$. The precision criteria shown are $\pm 5 \mathrm{msec}$ (filled squares) and $\pm 1 \mathrm{msec}$ (open squares).

output frequency and the input frequency (Fig. 6D) and between the output frequency and the membrane potential (Fig. 6E). Without input synchronization the output frequencies were zero for large input frequencies, and therefore, the frequency curves showed a threshold (Fig. 6D,E, triangles). Overall the spike frequency of DCN neurons can be regulated in a linear way by the frequency of inhibitory input neurons, and thus these neurons can be said to use a rate code in decoding the rate of Purkinje cell inputs.

The spike-timing precision of DCN neurons did not change for different inhibitory input frequencies (Fig. 6A,B), but it depended on the input gain and input synchronization (Fig. $6 C$ ). For all four conditions of gain and synchronicity, the proportion of stimulusaligned spikes for the $\pm 5 \mathrm{msec}$ precision criterion was independent of the input frequency (Fig. $6 C$ ). These results show that the frequency of DCN neurons can be regulated by the input frequency without affecting the spike-timing precision.

\section{The spike rate was strongly influenced by the input synchronization}

Although the spike rate was nearly linearly dependent on the input frequency for a given combination of input gain and synchronicity, it was quite different at the same input frequency for different levels of gain and synchronicity (Fig. 6D). Therefore spike-rate coding of DCN neurons does not simply reflect the rate of input events but also the pattern of inputs. To quantify this effect we calculated the frequency response and membrane potential of DCN neurons to our first stimulus set in which input gain and input synchronization were varied systematically (Fig. 7). We found that an increasing input gain led to a similar increase in membrane depolarization for intermediate and high input synchronization (Fig. 7A). As described above (Fig. 3) the depolarization with increasing gain is explained by a stronger voltage-clamp effect toward the synaptic reversal potential of -40 $\mathrm{mV}$ for high input gains. For a high level of input synchronicity this increase in depolarization was paralleled by an increase in spike rate (Fig. $7 B$ ). In contrast, an intermediate level of input synchronization led to a final decrease in spike rate with increasing input gain even though the neuron was overall more depolarized than with high input synchronization (Fig. 7A,B). A reduction in spike rate was found for all of the $21 \mathrm{DCN}$ neurons when input synchronicity was absent (Fig. 7C). A similar dependence between spike rate and input synchronization was seen in the experiments in which only one group of input elements was synchronized. Although the spike rate showed almost no dependence on the input synchronization at a gain of 2 , it increased linearly with an increasing number of synchronized inputs at a gain of 8 (Fig. 7D). These results can be explained by a different mechanism of triggering spikes when the input gain was low than when it was high. At low gains, spikes are initiated by intrinsic pacemaker activity based on the level of membrane depolarization. This corresponds to the case of current injection in vitro, in which DCN neurons show a nearly linear relationship between current-induced depolarization and output spike rate (Jahnsen, 1986a). At high input gains a fundamentally different mechanism determined the generation of spikes in our experiments. In this case intrinsic pacemaker currents could not determine the trajectory of $\mathrm{V}_{\mathrm{m}}$, because it was clamped to the level given by $\mathrm{V}_{\text {syn }}$ (Fig. $3 C, D)$. In the absence of large fluctuations in the input, spikes could not escape from the clamping potential even when the membrane was depolarized. Therefore, spike generation at high input gains required the presence of significant fluctuations in $\mathrm{V}_{\text {syn }}$. The amplitude of such fluctuations declined with a reduction of input synchronization (Fig. 4D), which led to a reduction in spike rate.

As argued above (Fig. 3) the condition of high input gain is expected to operate in vivo because low-gain stimuli do not show the irregular spike pattern recorded in the DCN of behaving 
A: voltage traces

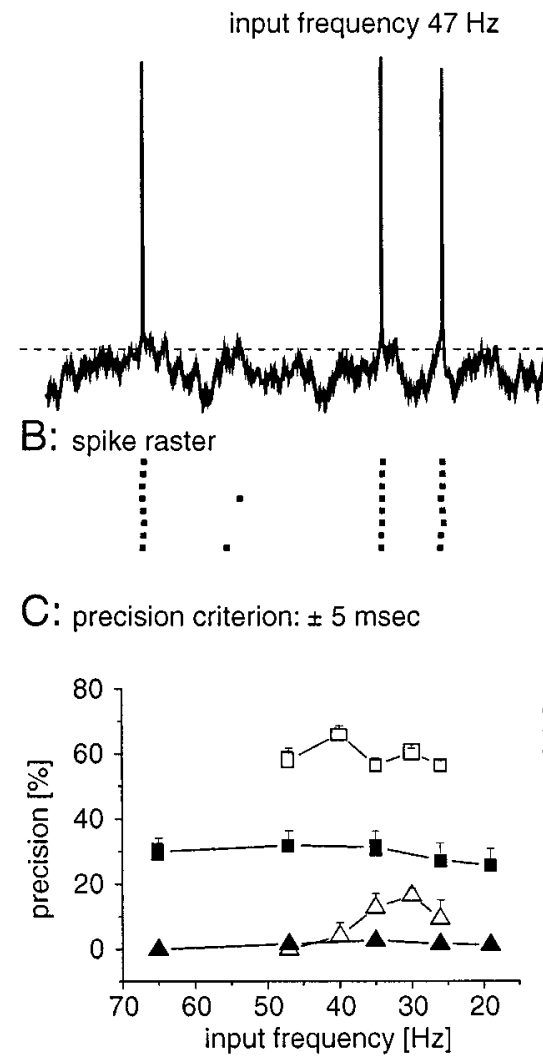

input frequency $26 \mathrm{~Hz}$

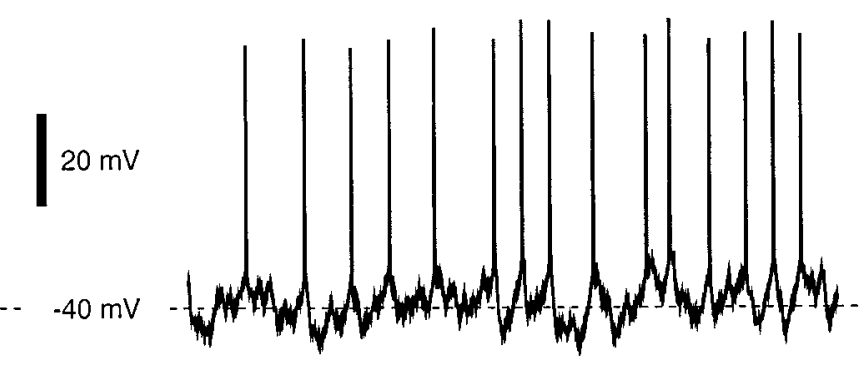

$200 \mathrm{msec}$

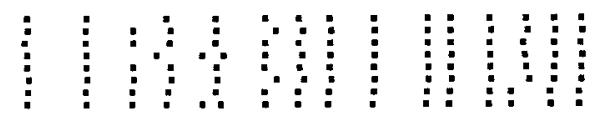

$E$

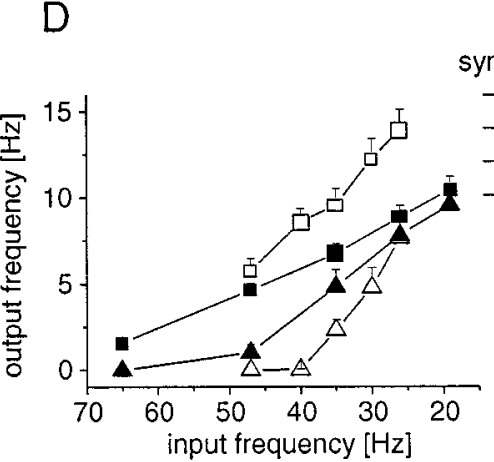

synchronization gain

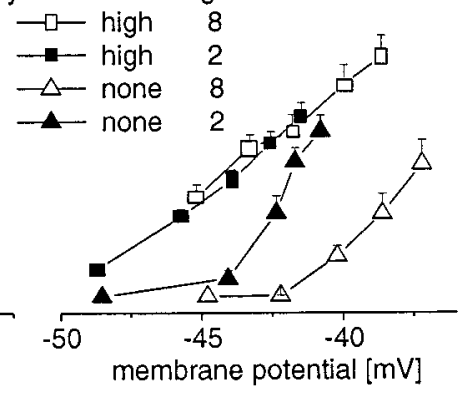

Figure 6. Input frequency determined output frequency but left spike-timing precision unaffected. $A$, Voltage traces are from one DCN neuron at 47 and $26 \mathrm{~Hz}$ of mean input frequency of each input element. The gain factor was 8 and the input synchronization was high for both input frequencies. $B$, Spike rasters of eight stimulus repetitions corresponding to the voltage traces in $A$ are shown. $C$, The spike-timing precision (criterion, \pm 5 msec) of five $\mathrm{DCN}$ (mean $\pm \mathrm{SE}$ ) neurons was independent from the frequency of the inhibitory input elements but depended on the input synchronization and input gain as shown in Figure 2. $D$, The mean spike frequency of the same neurons (output frequency) as a function of the input frequency of four stimulus conditions of gain and synchronization is shown. $E$, The output frequency of the same neurons as a function of $\mathrm{V}_{\mathrm{m}}$ is shown.

animals (Thach, 1968). We found that at the highest input gain the spike frequency was four times faster with high than without input synchronization (Fig. 7C). To achieve the same effect by a change in input frequency, an average reduction of Purkinje cell input rate by $>20 \mathrm{~Hz}$ was required (Fig. 6). Thus, changes in the synchronicity of inhibitory input can have as large an effect on the output spike rate as substantial changes in the total rate of input events.

\section{DISCUSSION}

\section{Rate and temporal coding via inhibitory input}

An important question in computational neuroscience is whether information might be encoded exclusively in the spike rate of neurons or in addition in the precise timing of spikes (Softky, 1995; Shadlen and Newsome, 1998). Current injection experiments illustrated that the spike timing of neurons can be controlled very precisely by temporally modulated currents (Mainen and Sejnowski, 1995). Information theoretical approaches indicate that a large amount of information about sensory inputs is potentially available in the precise spike timing (de Ruyter van Steveninck and Bialek, 1988; Borst and Theunissen, 1999). The discussion of possible mechanisms of a precise control of spike timing, however, has focused almost exclusively on synchronicity in excitatory inputs (Shadlen and Newsome, 1994, 1998; Holt et al., 1996; König et al., 1996). In the present study we examined whether and by what mechanisms inhibitory inputs may contribute to rate and temporal coding of output spikes. We found that synchronous activity and synchronous pauses in the input spike pattern resulted in the presence of transient decreases in the inhibitory conductance, which reliably and precisely triggered spikes. The spike rate of DCN neurons was strongly affected by the amount of synchronicity present in the input, and it depended also in an almost linear way on the rate of the inhibitory inputs. Thus, the spike rate of DCN neurons contained information about the spike rate as well as the synchronicity of inhibitory inputs, and the spike timing retained information about synchronous input events. A significant proportion of all presynaptic elements needed to be involved in synchronous activity patterns before they were effective. This is in contrast to a form of coincidence detection that has been postulated for excitation, in which strong dendritic nonlinearities would allow much smaller assembles of coactive inputs to trigger precisely timed spikes (Abeles, 1991; Aertsen et al., 1996; Holt et al., 1996). We used DCN neurons as an example to demonstrate possible coding by inhibition in a structure that is known to receive an important inhibitory projection pathway. Inhibitory projection pathways also occur outside the cerebellum, most notably in the basal ganglia. The effects shown, however, may also operate when a sufficient number of inhibitory inputs is received from local interneurons, as may be the case for pyramidal cortical cells. 

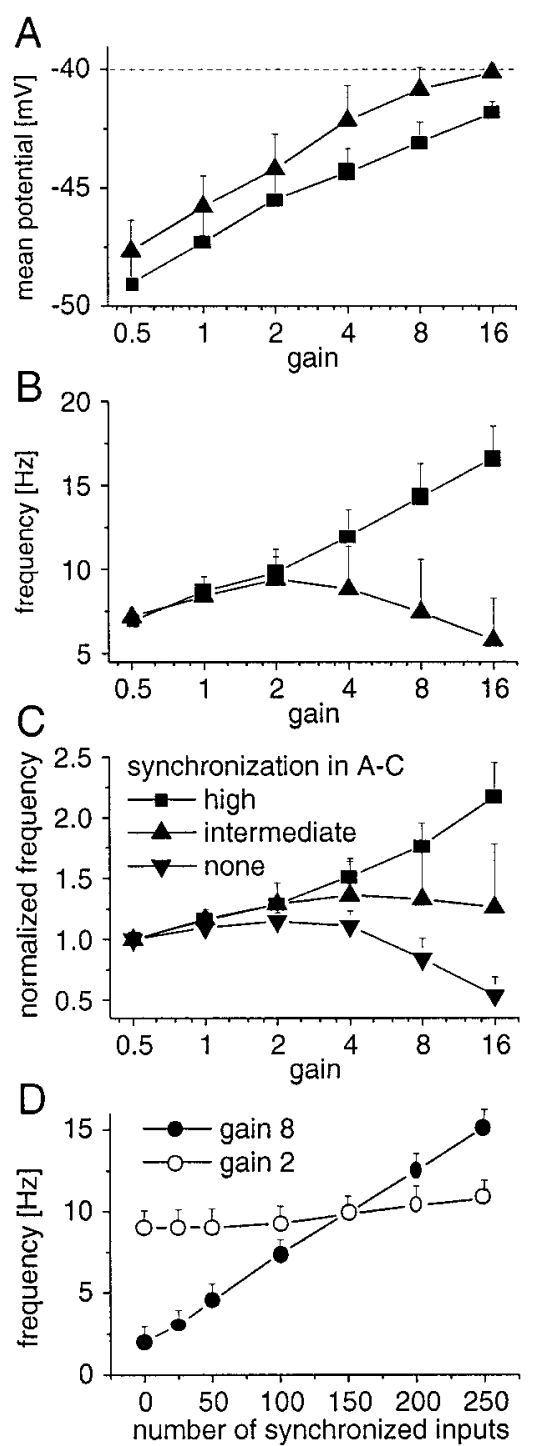

Figure 7. Spike frequency depended strongly on the input correlation at high input gains. $A, \mathrm{~V}_{\mathrm{m}}$ depolarized with increasing input gain at both input synchronizations tested. The data of one neuron calculated from six stimulus repetitions (mean $\pm \mathrm{SD}$ ) are shown. For each action potential a segment of $\mathrm{V}_{\mathrm{m}}$ was cut out $( \pm 3 \mathrm{msec})$ to isolate the subthreshold membrane depolarization. $B$, The spike frequency (mean $\pm \mathrm{SD}$ ) of the same traces examined in $A$ decreased after an initial increase for the intermediate input synchronization (triangles) despite an increasing $\mathrm{V}_{\mathrm{m}}(A)$. The frequency increased continuously for the highest input synchronization (squares). $C$, Spike frequencies of $21 \mathrm{DCN}$ neurons were normalized to a value of 1.0 at the input gain of 0.5 . The mean output frequencies at this gain were $10.0 \mathrm{~Hz}$ (high input synchronization), $9.0 \mathrm{~Hz}$ (intermediate synchronization), and $10.2 \mathrm{~Hz}$ (no synchronization). The SE for the intermediate synchronization was large at the highest gain, because three neurons did not show a decrease of spike frequency at this level of synchronization. $D$, Spike frequency as a function of input synchronization for a gain of 2 and 8 is shown. An increasing number of input elements in only one group was synchronized. The data are from the same six DCN neurons shown in Figure $2 E$.

\section{Inhibition can only act in balance with excitation}

We used simulated synaptic conductances as input patterns to DCN neurons in vitro to simulate an in vivo-like situation in which neurons receive thousands of synaptic inputs per second. This situation results in an ongoing baseline in the level of excitatory and inhibitory inputs, which provides a voltage clamp at the combined synaptic reversal potential $\left(\mathrm{V}_{\mathrm{syn}}\right.$; see Materials and Methods). The waveform of $\mathrm{V}_{\text {syn }}$ creates an attractor toward which the membrane potential is pulled with a force proportional to the clamp gain. We showed that at realistic input levels the actual membrane potential stayed relatively close to the command potential given by $\mathrm{V}_{\text {syn }}$. To achieve realistic spike rates, the value of $\mathrm{V}_{\text {syn }}$ in DCN neurons must be approximately $-40 \mathrm{mV}$, which dictates the required balance of excitation and inhibition.

DCN neurons receive excitatory inputs as collaterals from mossy and climbing fibers that also excite cerebellar cortex. These inputs are not likely to be constant over time as in our study and thus may control spike timing in addition to inhibitory input. A majority of excitatory inputs on DCN neurons, however, is located distal on dendrites (De Zeeuw and Berrebi, 1995), which is likely to attenuate fast transients. Furthermore, excitatory inputs have a large NMDA component even at negative membrane potentials (Anchisi et al., 1998), which leads to a prolonged effect of excitation. These characteristics suggest that coincident excitatory input events might not be very effective in the rapid control of single spikes but may be important on a slower timescale, perhaps in conjunction with depolarized dendritic plateau states. The presence of active dendritic conductances that could lead to an amplification of fast AMPA inputs cannot be excluded at the present time, however. The actual dynamics of the excitatory input and their interaction with intrinsic properties may form an important component of DCN function and remain to be determined.

\section{Contribution of intrinsic conductances to the control of spiking}

We found that the voltage-clamp effect of large numbers of synaptic inputs effectively controls the subthreshold membrane potential. Although this effect to some degree shunts voltagegated currents present in the neuron, their activation can still have a profound influence on the observed spike pattern. This is apparent when different cell types are stimulated with the same synaptic conductance patterns, and characteristically different output spike patterns are obtained (D. Jaeger, unpublished observations). A significant influence on the spike pattern is likely given by the voltage dependence and time constants of the depolarizing spike current itself, which needs to escape from $\mathrm{V}_{\text {syn }}$ before any spike can occur. DCN neurons also have a persistent Na current (Gardette et al., 1985; Jahnsen, 1986b), which likely contributes to depolarizing the cell to spike initiation (Koch et al., 1995; Crill, 1996). Other subthreshold currents are also important, however. In DCN neurons a substantial hyperpolarizing current is activated after each action potential (Jahnsen, 1986b; Aizenman and Linden, 1999). In our experiments this current was partly counteracted by a depolarizing synaptic-clamp current (Fig. 3D), but nevertheless it led to a significant postspike hyperpolarization (Figs. $1 B, 3 C$ ). This hyperpolarization limited the spike frequency during depolarized phases of $\mathrm{V}_{\text {syn }}$. Another potential current affecting the spike pattern of DCN neurons is the low-threshold calcium current, which could lead to postinhibitory rebound spiking (Llinás and Mühlethaler, 1988; Aizenman et al., 1998; Aizenman and Linden, 1999). In our experiments the contribution of this current was likely to be minimal, however, because the prolonged periods of membrane hyperpolarization required to deinactivate this current were not present.

\section{Spike irregularity and the gain of input conductances}

Irregular spike patterns are found in many brain structures, and the debate has been lively for several years whether they reflect 
coincidence detection within a temporal code (Softky and Koch, 1993; Softky, 1995) or whether they represent randomness within a rate code (Shadlen and Newsome, 1994, 1998). Softky and Koch (1993) suggested that excitatory inputs could cause irregular firing only when the input synchronicity is large or when strong dendritic nonlinearities exist. We found that inhibition can cause irregularity in the spike pattern as recorded in DCN neurons in vivo (Thach, 1968) for all levels of input synchronization tested when the input gain was high. At high input gains, the total synaptic conductance was strong enough to overcome the intrinsic pacemaker dynamics of the neuron and to clamp $V_{m}$ close to $V_{\text {syn }}$. Therefore, fluctuations in $\mathrm{V}_{\text {syn }}$ determined the timing of spike threshold crossings. At low or high levels of input synchronization fluctuations that led to threshold crossings in $\mathrm{V}_{\mathrm{m}}$ were irregular, but at a reduced input synchronization they occurred less frequently, and the spike rate was reduced. Random small fluctuations in the input cannot add up in this system because $\mathrm{V}_{\mathrm{m}}$ is continuously forced toward $\mathrm{V}_{\text {syn }}$. Thus, the voltage-clamp control of spiking we observed is not compatible with the view of synaptic current creating a "random walk," as proposed by Shadlen and Newsome $(1994,1998)$. Also in contrast to the model of Shadlen and Newsome, the membrane time constant does not determine the input integration time constant. Instead, the time required to depolarize $\mathrm{V}_{\mathrm{m}}$ to trigger a spike at a high input gain closely followed the time course of $\mathrm{V}_{\text {syn }}$.

The presence of a large voltage-clamp gain in other brain structures is supported by in vivo intracellular data showing that fluctuations in subthreshold membrane potential are rapid and of large amplitude in cortical pyramidal neurons as well as other cell types (Paré et al., 1998; Stern et al., 1998; Lampl et al., 1999). The large synaptic current required to drive such changes in $V_{m}$ indicates the presence of large synaptic conductances and hence the operation of a synaptic voltage-clamp mechanism at high gain as described.

\section{The control of DCN spiking by Purkinje cell input}

Many investigators have linked the function of the cerebellum to fine temporal aspects of movement control (Braitenberg, 1967; Ivry and Keele, 1989; Houk et al., 1996; Braitenberg et al., 1997; Mauk et al., 1998; Wang et al., 1998; Timmann et al., 1999). Such control requires that the output from the DCN is temporally precise on the order of a few milliseconds. Furthermore, the DCN consist of relatively few neurons for each effector system such that a mechanism of averaging the spike rate of a large population of DCN neurons to control movement timing seems unlikely. Therefore spike trains of a small number of DCN neurons need to be sufficient to transmit temporally precise information. Recordings from DCN neurons in relation to saccades show indeed that the temporal alignment of DCN spiking to external events can be at least 5 msec (Takikawa et al., 1998). Our data show that inhibitory Purkinje cell input is suitable to control DCN spiking at this precision, if synchronous activation changes are present in a large population of Purkinje cell inputs. This requirement places constraints on the computation taking place in cerebellar cortex, because only events that influence substantial numbers of Purkinje cells appear to be significant. The exact geometrical layout of the convergence pattern of Purkinje cells onto single DCN neurons is still unknown. If distinct patches or sagittal stripes of Purkinje cells were to contact single DCN neurons, further inferences about cerebellar computation could be drawn. For example, the tidal wave theory by Braitenberg et al. (1997) would require that Purkinje cells placed along a parallel fiber bundle project to the same DCN neurons. In contrast, the model of coactivated patches of Purkinje cells (Bower, 1997) would predict a convergent projection of patches of Purkinje cells onto single DCN neurons.

\section{REFERENCES}

Abeles M (1991) Corticonics: neural circuits of the cerebral cortex. New York: Cambridge UP.

Aertsen A, Diesmann M, Gewaltig MO (1996) Propagation of synchronous spiking activity in feedforward neural networks. J Physiol (Paris) 90:243-247.

Aizenman CD, Linden DJ (1999) Regulation of the rebound depolarization and spontaneous firing patterns of deep nuclear neurons in slices of rat cerebellum. J Neurophysiol 82:1697-1709.

Aizenman CD, Manis PB, Linden DJ (1998) Polarity of long-term synaptic gain change is related to postsynaptic spike firing at a cerebellar inhibitory synapse. Neuron 21:827-835.

Anchisi D, Scelfo B, Strata P, Tempia F (1998) Postsynaptic currents in deep cerebellar nuclei. Soc Neurosci Abstr 24:325.

Barry PH (1994) JPCalc, a software package for calculating liquid junction potential corrections in patch-clamp, intracellular, epithelial and bilayer measurements and for correcting junction potential measurements. J Neurosci Methods 51:107-116.

Barry PH, Lynch JW (1991) Liquid junction potentials and small cell effects in patch-clamp analysis. J Membr Biol 121:101-117.

Batini C, Compoint C, Buisseret-Delmas C, Daniel H, Guegan M (1992) Cerebellar nuclei and the nucleocortical projections in the rat: retrograde tracing coupled to GABA and glutamate immunohistochemistry. J Comp Neurol 315:74-84.

Bialek W, Rieke F (1992) Reliability and information transmission in spiking neurons. Trends Neurosci 15:428-434.

Billard JM, Vigot R, Batini C (1993) GABA, THIP and baclofen inhibition of Purkinje cells and cerebellar nuclei neurons. Neurosci Res 16:65-69.

Borst A, Theunissen FE (1999) Information theory and neural coding. Nat Neurosci 2:947-957.

Bower JM (1997) Is the cerebellum sensory for motor's sake, or motor for sensory's sake: the view from the whiskers of a rat? Prog Brain Res 114: 483-516.

Braitenberg V (1967) Is the cerebellar cortex a biological clock in the millisecond range? Prog Brain Res 25:334-346.

Braitenberg V, Heck D, Sultan F (1997) The detection and generation of sequences as a key to cerebellar function: experiments and theory. Behav Brain Sci 20:229-277.

Crill WE (1996) Persistent sodium current in mammalian central neurons. Annu Rev Neurosci 58:349-362.

de Ruyter van Steveninck R, Bialek W (1988) Real-time performance of a movement-sensitive neuron in the blowfly visual system: coding and information transfer in short spike sequences. Proc R Soc Lond [Biol] 234:379-414.

De Zeeuw CI, Berrebi AS (1995) Postsynaptic targets of Purkinje cell terminals in the cerebellar and vestibular nuclei of the rat. Eur J Neurosci 7:2322-2333.

Diener HC, Hore J, Ivry R, Dichgans J (1993) Cerebellar dysf unction of movement and perception. Can J Neurol Sci 20[Suppl 3]:S62-S69.

Fortier PA, Smith AM, Kalaska JF (1993) Comparison of cerebellar and motor cortex activity during reaching: directional tuning and response variability. J Neurophysiol 69:1136-1149.

Gardette R, Debono M, Dupont J-L, Crepel F (1985) Electrophysiological studies on the postnatal development of intracerebellar nuclei neurons in rat cerebellar slices maintained in vitro. II. Membrane conductances. Dev Brain Res 20:97-106.

Häusser M, Clark BA (1997) Tonic synaptic inhibition modulates neuronal output pattern and spatiotemporal synaptic integration. Neuron 19:665-678.

Holmes WR, Woody CD (1989) Effects of uniform and non-uniform synaptic "activation-distributions" on the cable properties of modeled cortical pyramidal neurons. Brain Res 505:12-22.

Holt G, Softky WR, Koch C, Douglas RJ (1996) Comparison of discharge variability in vitro and in vivo in cat visual cortex neurons. J Neurophysiol 75:1806-1814.

Houk JC, Buckingham JT, Barto AG (1996) Models of the cerebellum and motor learning. Behav Brain Sci 19:368-383. 
Ivry RB, Keele SW (1989) Timing functions of the cerebellum. J Cognit Neurosci 1:136-152.

Jaeger D, Bower JM (1999) Synaptic control of spiking in cerebellar Purkinje cells: dynamic current clamp based on model conductances. J Neurosci 19:6090-6101.

Jahnsen H (1986a) Electrophysiological characteristics of neurones in the guinea-pig deep cerebellar nuclei in vitro. J Physiol (Lond) 372:129-147.

Jahnsen H (1986b) Extracellular activation and membrane conductances of neurones in the guinea-pig deep cerebellar nuclei in vitro. J Physiol (Lond) 372:149-168.

Koch C, Bernander O, Douglas RJ (1995) Do neurons have a voltage or a current threshold for action potential initiation? J Comput Neurosci 2:63-82.

König P, Engel AK, Singer W (1996) Integrator or coincidence detector? The role of the cortical neuron revisited. Trends Neurosci 19:130-141.

Krauzlis RJ, Lisberger SG (1996) Directional organization of eye movement and visual signals in the floccular lobe of the monkey cerebellum. Exp Brain Res 109:289-302.

Lampl I, Reichova I, Ferster D (1999) Synchronous membrane potential fluctuations in neurons of the cat visual cortex. Neuron 22:361-374.

Lang EJ, Sugihara I, Welsh JP, Llinás R (1999) Patterns of spontaneous Purkinje cell complex spike activity in the awake rat. J Neurosci 19:2728-2739.

LeDoux MS, Hurst DC, Lorden JF (1998) Single-unit activity of cerebellar nuclear cells in the awake genetically dystonic rat. Neuroscience $86: 533-545$.

Llinás R, Mühlethaler M (1988) Electrophysiology of guinea-pig cerebellar nuclear cells in the in vitro brain stem-cerebellar preparation. J Physiol (Lond) 404:241-258.

Mainen ZF, Sejnowski TJ (1995) Reliability of spike timing in neocortical neurons. Science 268:1503-1506.

Mano N, Ito Y, Shibutani H (1991) Saccade-related Purkinje cells in the cerebellar hemispheres of the monkey. Exp Brain Res 84:465-470.

Mauk MD, Garcia KS, Medina JF, Steele PM (1998) Does cerebellar LTD mediate motor learning? Toward a resolution without a smoking gun. Neuron 20:359-362.

Palkovits M, Mezey E, Hamori J, Szentagothai J (1977) Quantitative histological analysis of the cerebellar nuclei in the cat. I. Numerical data on cells and synapses. Exp Brain Res 28:189-209.

Paré D, Shink E, Gaudreau H, Destexhe A, Lang EJ (1998) Impact of spontaneous synaptic activity on the resting properties of cat neocortical pyramidal neurons in vivo. J Neurophysiol 79:1450-1460.

Paulin MG (1996) System identification of spiking sensory neurons using realistically constrained nonlinear time series models. In: Advances in processing and pattern analysis of biological signals (Gath I, Inbar G, eds). New York: Plenum.

Rapp M, Yarom Y, Segev I (1992) The impact of parallel fiber background activity on the cable properties of cerebellar Purkinje cells. Neural Comput 4:518-533.

Rieke F, Warland D, de Ruyter van Steveninck R, Bialek W (1996) Spikes. Exploring the neural code. Cambridge, MA: MIT.

Robinson HPC, Kawai N (1993) Injection of digitally synthesized synaptic conductance transients to measure the integrative properties of neurons. J Neurosci Methods 49:157-165.

Savio T, Tempia F (1985) On the Purkinje cell activity increase induced by suppression of inferior olive activity. Exp Brain Res 57:456-463.

Shadlen MN, Newsome WT (1994) Noise, neural codes and cortical organization. Curr Opin Neurobiol 4:569-579.

Shadlen MN, Newsome WT (1998) The variable discharge of cortical neurons: implications for connectivity, computation, and information coding. J Neurosci 18:3870-3896.

Sharp AA, O'Neil MB, Abbott LF, Marder E (1993) Dynamic clamp: computer-generated conductances in real neurons. J Neurophysiol 69:992-995.

Softky WR (1995) Simple codes versus efficient codes. Curr Opin Neurobiol 5:239-247.

Softky WR, Koch C (1993) The highly irregular firing of cortical cells is inconsistent with temporal integration of random EPSPs. J Neurosci 13:334-350.

Stern EA, Jaeger D, Wilson CJ (1998) Membrane potential synchrony of simultaneously recorded striatal spiny neurons in vivo. Nature 394:475-478.

Stratton SE, Lorden JF, Mays LE, Oltmans GA (1988) Spontaneous and harmaline-stimulated Purkinje cell activity in rats with a genetic movement disorder. J Neurosci 8:3327-3336.

Takikawa Y, Kawagoe R, Miyashita N, Hikosaka O (1998) Presaccadic omnidirectional burst activity in the basal interstitial nucleus in the monkey cerebellum. Exp Brain Res 121:442-450.

Thach WT (1968) Discharge of Purkinje and cerebellar nuclear neurons during rapidly alternating arm movements in the monkey. J Neurophysiol 31:785-797.

Timmann D, Watts S, Hore J (1999) Failure of cerebellar patients to time finger opening precisely causes ball high-low inaccuracy in overarm throws. J Neurophysiol 82:103-114.

Wang JJ, Shimansky Y, Bracha V, Bloedel JR (1998) Effects of cerebellar nuclear inactivation on the learning of a complex forelimb movement in cats. J Neurophysiol 79:2447-2459.

Welsh JP, Lang EJ, Sugihara I, Llinás R (1995) Dynamic organization of motor control within the olivocerebellar system. Nature 374:453457. 